\title{
Improving the Raster Scanning Methods used with X-Ray Fluorescence to see the Ancient Greek Text of Archimedes
}

\author{
Isabella B. Griffin \\ Office of Science, SULI Program \\ Norfolk Sate University \\ Stanford Linear Accelerator Center \\ Menlo Park, California
}

August 20, 2005

Prepared in partial fulfillment of the requirements of the Office of Science Undergraduate Laboratory Internship (SULI) Program under the direction of Dr. Uwe Bergmann of Stanford Synchrotron Radiation Laboratory.

Participant:

$$
\text { Signature }
$$

Research Advisor:

$$
\text { Signature }
$$

Any disclaimer(s) required by your host DOE laboratory (if any). 


\section{Table of Contents}

Abstract....................................................ii

Introduction..............................................

Lab Setup and Equipment................................

Results and Discussion................................ 7

Conclusion.......................................................

Acknowledgements...................................11

References.............................................12

Tables.....................................................12

Formulas................................................13

Figures...............................................13 


\section{ABSTRACT: \\ Improving the raster scanning methods used with $x$-ray fluorescence to see the ancient Greek copy of Archimedes work. ISABELLA GRIFFIN (Norfolk State University, Norfolk Virginia 23504) DR. UWE BERGMANN (Stanford Synchrotron Radiation Laboratory, Menlo Park California)}

X-ray fluorescence is being used to detect the ancient Greek copy of Archimedes work. The copy of Archimedes text was erased with a weak acid and written over to make a prayer book in the Middle Ages. The ancient parchment, made of goat skin, has on it some of Archimedes most valuable writings. The ink in the text contains iron which will fluoresce under x-ray radiation.

My research project deals with the scanning and imaging process. The palimpsest is put in a stage that moves in a raster format. As the beam hits the parchment, a germanium detector detects the iron atoms and discriminates against other elements. Since the computer scans in both forwards and backwards directions, it is imperative that each row of data lines up exactly on top of the next row.

There are several parameters to consider when scanning the parchment. These parameters include: speed, count time, shutter time, x-number of points, and acceleration. Formulas were made to relate these parameters together. During the actual beam time of this project, the scanning was very slow going; it took 30 hours to scan $1 / 2$ of a page. Using the formulas, the scientists doubled distance and speed to scan the parchment faster; however, the grey scaled data was not lined up properly causing the images to look blurred. My project was is to find out why doubling the parameters caused blurred images, and to fix the problem if it is fixable. 


\section{Introduction}

Archimedes was a revolutionary scholar and was centuries ahead of his time. He is responsible for approximating pi, discovering the principles on buoyancy, and was the first to use premature forms of calculus to solve problems. In the $12^{\text {th }}$ century, a $10^{\text {th }}$ century Greek copy of Archimedes writings was erased with a weak acid, like lemon juice, and written over by a Christian monk to make a prayer book. In the middle ages, parchment was very expensive and hard to come by, therefore when parchment was reused the resulting book was called a palimpsest [1]. The pages of the Archimedes parchment were folded horizontally and bound together so that the Archimedes text lays perpendicular to that of the prayers. These particular writings of Archimedes are very important because they contain the only copy of Archimedes' Method of Mechanical Theorems, in which Archimedes explains how he came up with his theorems. This is also the only document that contains original diagrams of Archimedes [1].

The palimpsest was discovered in 1906 by Johan Ludvig Heiberg in Constantinople, present day Istanbul [1]. He took pictures of the pages because he could not remove the palimpsest from the library. He knew it was the lost Archimedes writings, but he did not have the technology to study it in great detail. After WWI, the palimpsest was lost, not to resurface again till the 1990's in France [1]. The palimpsest was sold on auction by Christies to an anonymous billionaire for $\$ 2$ million. The new owner sent it to Walters Art Museum in Baltimore for further study and restoration [1].

Most of the text has been recovered using powerful cameras and special lenses in visible or ultra-violet light and a process known as multi-spectral imaging. This process uses different light of different wavelengths to distinguish between the two different texts 
[1]. However, $20-30 \%$ of the text could still not been read. Some of the text is too faint, some covered by mold or other obstacles, and four of the pages have forged paintings on them. The big problem was how to get past the paint to see the text. This is when Stanford linear Accelerator Center’s (SLAC) Stanford Synchrotron Radiation Laboratory (SSRL) comes into play. The ink used to write Archimedes work contains iron. When put under the x-ray radiation, the iron atoms will fluoresce, or glow, allowing scientist to see beneath the paint and to the original Archimedes text. The work was published in a press release from the journal Nature, SLAC, and from the Walters Art Museum causing enormous media attention (see reference 4).

X-ray beams from SSRL were used to see the hidden text. In a synchrotron, electrons, moving at close to the speed of light, are accelerating in a storage ring. The acceleration causes the electrons to give off $\mathrm{x}$-ray radiation

The ink in the Archimedes text contains iron, which will fluoresce when excited. When an x-ray beam hits an iron atom the x-ray can be absorbed or scattered. If it is absorbed it undergoes a process called the photoelectric effect [2]. In this process, if the x-ray beam has enough energy, electrons in the inner shells are knocked out creating electron holes. These holes put the atom into an excited state. In order to return into the ground state the electrons from the outer shells quickly fill the vacant spots. In doing so, these electrons give off $x$-rays [2]. Electrons in different energy levels have different binding energies. The energy of the $\mathrm{x}$-rays emitted is the difference in the two binding energies of the shells to and from which the electron jumped. Each element has a characteristic x-ray because each element has a unique set of energy levels. X-ray 
fluorescence is the process in which one evokes the emission of the characteristic x-rays known to an element [2].

The pages were put on a stage that moves in the $\mathrm{x}$ and $\mathrm{y}$ direction on a raster format. As the small $\mathrm{x}$-ray beam hits the page, a germanium detector will detect the fluorescence of the iron and discriminate against that of other elements.

My part in this project is with the scanning procedure. The current problem with our scanning procedure is to synchronize the read out with the scanning speed.

Furthermore, we have a dead time at the end of each readout that limits the ultimate imaging speed. I will show experiments that help to improve the scanning procedure's efficiency for future use.

\section{Lab Setup and Equipment}

Because my project was to improve the scanning procedure, the original Archimedes Palimpsest and the x-ray beam were not needed. We substituted a class II He Ne laser for the x-ray beam and a photodiode detector instead of the original germanium detector. With this detector we measure the transmission of the laser beam through a selfmade stencil. The stencil was made by using a thin, yet sturdy type of cardboard. On it, I cut thin slits in the shape of old English script. I mounted the scroll on a frame that slides into the stage. The laser and detector are lined up with each other so that the laser beam goes directly into the pinhole of the detector. The scroll is scanned on a raster format. When the laser gets to a cutout, the photodiode detector will receive the beam. I scanned the stencil for about 2 weeks, but I was not able to produce a perfectly lined up image. I attributed this to the flaws in my cutout. I then scanned calipers. The advantages with the calipers were they have straight edges, and I was able to control the width of the line. I 
then made a transparency with old English text printed on it. The transparency was used to scan full text images.

\section{The Detector}

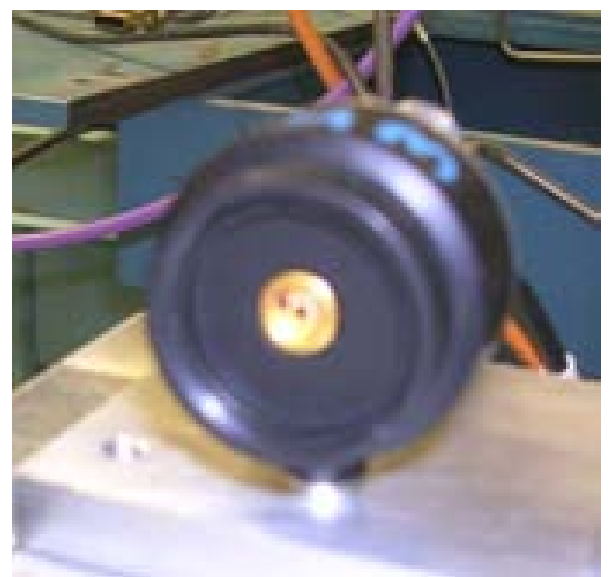

Image 1 : The photodiode detector was mounted behind The shutter box, which was removes from the image.

The detector I used in my experiment was a photodiode detector operating in

current mode. It sends out a current, that is fed into an amplifier which turns it into a voltage. The voltage signal is then changed into a frequency by a voltage to frequency converter. The frequency is converted into a numerical value when performing a scan. In order to show the image, these numerical numbers are then turned into a grey scale image using a grey scaling program, Rasplot, which will produce an image based on the numerical data. The detector used in the real experiment was a germanium detector. 


\section{The Shutter Box}

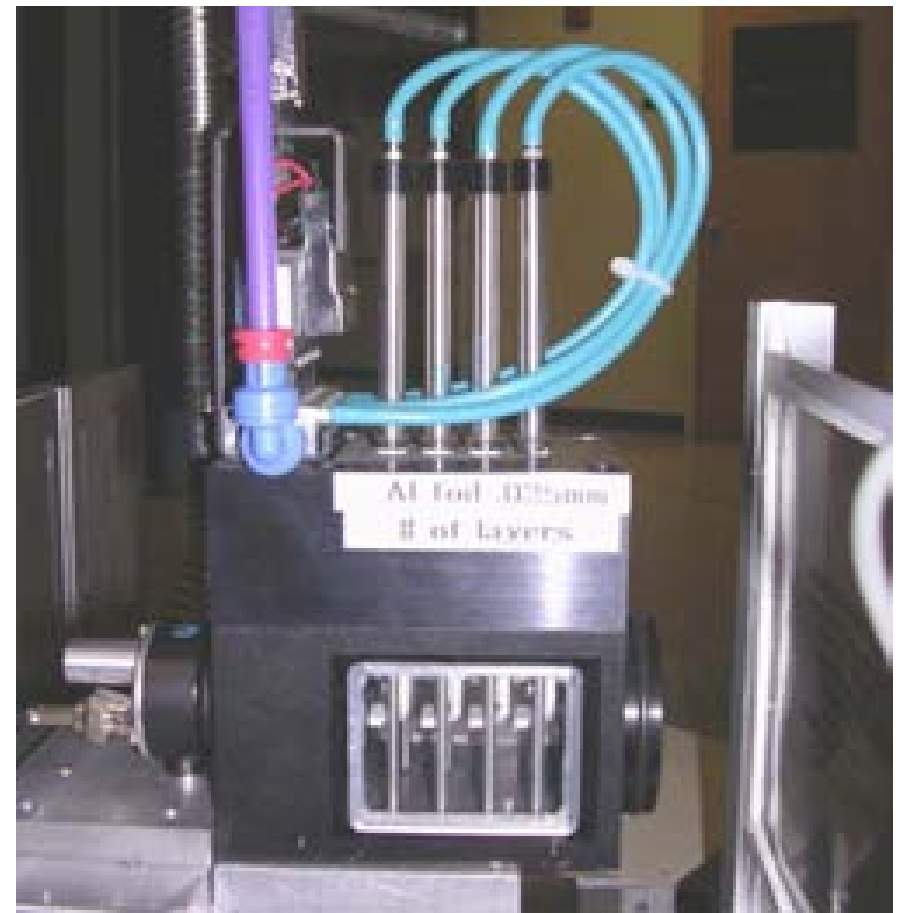

Image 2: The shutter box uses air pressure (in the blue tubes) to open and close the shutters.

The shutter box is used to protect the parchment from radiation damage caused by

the x-ray beam. The shutter stays open while the document is scanning, but when the stage gets to the end of the line, the shutter closes and the stage moves to the next line. When the shutter opens again, a new line is scanned. Although the beam is only at the end of the line for a split second, the radiation can still do damage to it. The shutter box will prevent the x-ray beam from being on the endpoints for too long. Formula 1 shows how to calculate the shutter time for a scan. 


\section{The Stage and Motors}

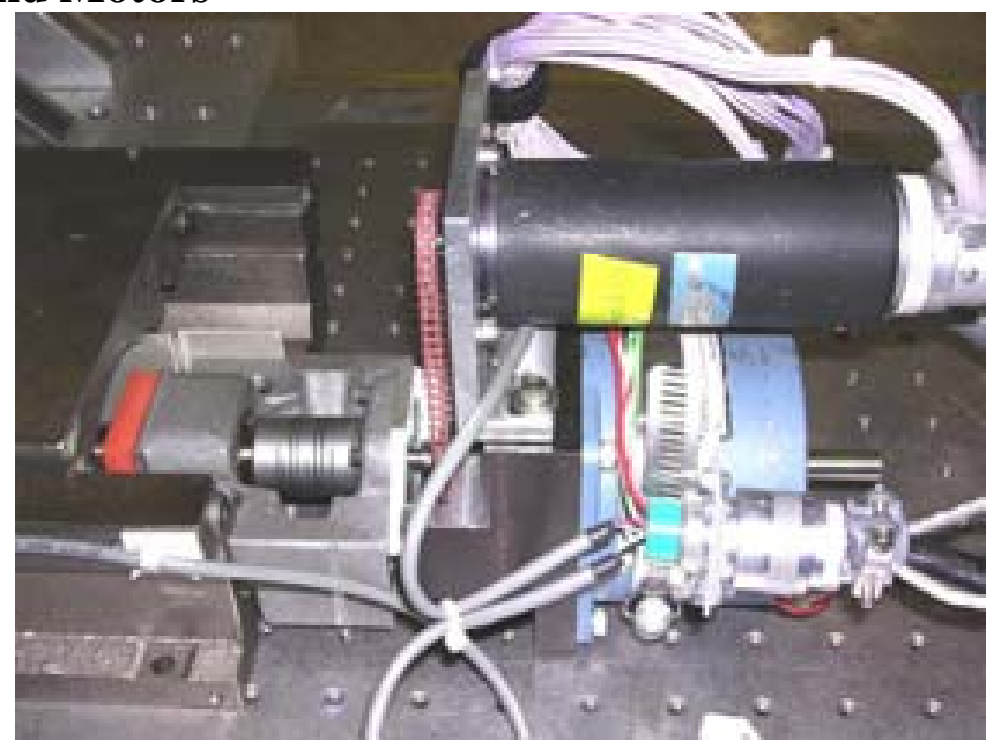

Image 3: (above) The stepper motor for the x-direction

Image 4: (below) The experimental set up; the laser, stage, shutter box, and detector

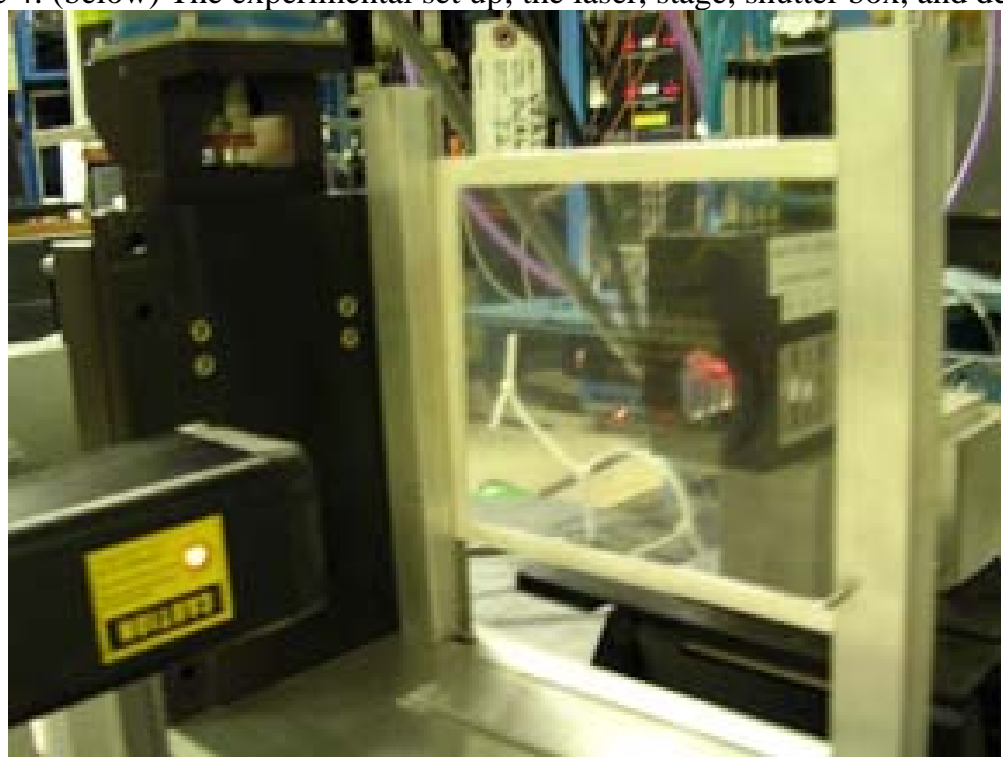

The type of motor used to scan is a stepper motor. 400 steps is one full revolution of the screw or equal to $2.54 \mathrm{~mm}$ (1/10 inch) of scan distance. Therefore 157.48 steps 
correspond to $1 \mathrm{~mm}$. There were two motors used to scan; one for the x-direction, the other for the $y$-direction. The $x$-motor was set to take 100 steps to accelerate to the desired speed, then 100 steps to decelerate to a stop. I hypothesized that the misalignment of the images could be due to a non-constant speed between intervals of acceleration and deceleration. This question prompted me to perform an experiment to see if the speed was in fact "tailing off”, which I will discuss later in the paper.

\section{Drivers}

I also experimented with different drivers. The original driver used in the experiment was an E500. This driver has been a standard at SSRL for a decade. It is relatively outdated equipment. I switched to a VXI driver and took scans with it for a few days. The VXI driver is a newer technology. The VXI driver did not produce any clear images, so I switched back to the E500.

\section{Results and Discussion}

When the original Archimedes Palimpsest was at SSRL, there were a standard set of parameters used for the x scans. These parameters are: count time, distance, speed, shutter time, and x-number of points (pixels). The count time is simply the time it takes for the parchments to move between readouts. Consequently, the distance the parchment travels during that time corresponds to the pixel size. A pixel size of $40 \mu[\sim 600$ dpi (dots per inch)] was used, and the actual size of the x-ray beam was slightly larger $(50 \mathrm{~m}$ diameter). The shutter time is the time period in which the shutter is open (see Formula 1) when scanning one full line. The x-number of points is how many $40 \mu$ pixels are in the desired scanning distance. These parameters relate to each other by a series of formulas (see Formulas Section). Since line scans where taken in both directions, if the timing 
between readout time and scanning speed is not perfect, subsequent lines do not line up properly, thus resulting in a blur or even a ghost image. The original parameters used a count time of $0.01 \mathrm{~ms}$, a distance of $20 \mathrm{~mm}$, a shutter time of $6.12 \mathrm{sec}$, and a speed of 494 steps/sec ( $3.14 \mathrm{~mm} / \mathrm{sec})$. This resulted in a clear image where subsequent $\mathrm{x}$-lines were aligned. The corresponding time to scan one half of the page of the palimpsest is approximately 30 hours. But it was for example found that doubling the speed did not result in good images and even a change in scan range introduced misalignments. In order to optimize the scanning procedures that result in clear images where subsequent lines are not offset to each other, it was my job to first find out why doubling the parameters did work, and to then produce a clear image with varying distances and speeds.

My first task was to reproduce a clear image using the original parameters from the Archimedes scans. I ran scans with the original parameters and was able to produce a good image (see Figure 1). However, the image showed me that I hadn’t been able to cut a straight line. I then replaced my stencil with a set of calipers (see Figure 2). The advantage with the calipers was not only the straight edge but the ability to control the width of the line. In order to scan the calipers I had to change the distance because the width of the calipers was much smaller than that of the stencil. Using the original formula, I changed the distance to $15 \mathrm{~mm}, \mathrm{x}$-number of points $=375$, count time $=.01$, shutter time $=4.51$, speed $=494$, acceleration $=100$ steps to fit the calipers, but subsequent lines were offset. This was when I first notice that the formulas were not quite correct. However, changing the speed slightly to 496 produced a clear image. 
I then attempted to produce another lined up image with a distance of $40 \mathrm{~mm}$. I was able to do that using the following parameters: distance $=40 \mathrm{~mm}$, $\mathrm{x}$-number of points $=1000$, count time $=.01$, shutter time $=12.45$, speed $=496$, acceleration $=100$ steps . Next I tried to scan half the distance using half the speed. I was unsuccessful. When experimenting with different count times, speeds, and accelerations, I was still unable to get a clear image. As it turned out the problem was related to the software as will be discussed below.

In order to test if one reason for misaligned images was related to a non-constant speed, I devised an experiment called the "speed test". In it, I scanned small distances (i.e. $10 \mathrm{~mm}$ ) and set the acceleration from 100 steps to 10 steps. We hypothesized that if the speed is gradually slowing down, then a scan of a short distance would look significantly better than a scan of a longer distance. I tested a $10 \mathrm{~mm}$ distance in great detail in an attempt to get a clear image. I did not get an image that was significantly better than the previous images. Amore crucial test is to vary the distances and see which distance produced the best image. This led me to conclude that the speed was constant between intervals of acceleration and deceleration. As an independent test, we hooked up an oscilloscope to both the E500 and the VXI to view the motion of the motors. At this point we changed the acceleration for 100 to10. We used a faster acceleration so we could have longer periods of constant speed. Both drivers produced the same results: the speed was constant between intervals of acceleration and deceleration. Figure 3 shows a graph of the motion of the motors.

I then tried to scan an image with a faster speed (815) and twice the distance $(40 \mathrm{~mm})$ of the original parameters. The image did not line up at all, and it was at this 
point when we realized that the counting time was not corresponding to the input. My part time mentor, Martin George, wrote a program that checks the count time of each scan. After using this program on several scans, we found out that the count time was not working on the computer. It did not count faster than $0.01 \mathrm{sec}$ even if another value was entered into the computer.

Since the count time did not work, the only way to line up the images at a faster was to change the x-number of points, hence the resolution. With this knowledge, I was able to find parameters that scanned clear images. In Table 1 the different distances, speeds, and x-number of points that produce clear images are summarized.

After taking over 250 scans in the past 4 weeks, I continued scanning with the E500 and have been able to find, for different distances, the speeds and x-number of points that will give lined up images. The distances I tested were $10 \mathrm{~mm}, 20 \mathrm{~mm}, 40 \mathrm{~mm}$, 60mm, and 80mm (see Table 1). After I found the correct parameters for each distance I scanned real text on a transparency that I made. I printed Old English text with a high quality printer in transparency paper. I made a frame for it and put it in the stage. I scanned this image when I found a new set of parameters that gave me a straight line after scanning the calipers. I used the transparency to see how well the image lined up over long distances. Figures 4 (before optimizing) and 5 ( optimized parameters) show images from the transparency scanned over a distance of $80 \mathrm{~mm}$. I furthermore learned about the mechanical limits of the scanning stage. During one scan a heavy liquid nitrogen dewar was pushed across the experimental floor and an image taken at that time showed a clear degradation in quality. We attributed this to possible vibrations caused by the moving dewar. 


\section{Conclusion}

At the end of eight weeks, and after nearly 300 scans, I have gained sufficient knowledge of the scanning equipment and procedures in the Archimedes Project. The reason why doubling the parameters did not work was because the count time was at a fixed 0.01 seconds. Unfortunately with the current software shorter readout times cannot be achieved. Faster scans of longer distances can still be accomplished if the pixel size is enlarged. However, varying the pixel size will compromise the resolution. To maintain 600 dpi resolution the standard speed of 496steps/mm must be kept, but the scan distances can now be changed and still produce clear images. I also found that using the formula to find the $x$-number of points and adding an imperially found 7 to the final number (see formula 3) will give the right $\mathrm{x}$-number of points for the corresponding distance. This additional offset is most likely caused by the acceleration and deceleration that had not previously been taken into account for. The revised formula will now allow us to vary the $\mathrm{x}$-distance without the loss of image quality. This will be very helpful for further imaging when in the Archimedes palimpsest returns to SSRL in early 2006. In the future, it is planned to replace the readout system in order to minimize the dead time and speedup the readout time. This will allow us to perfume faster scans without loss of resolution.

\section{Acknowledgements}

I would like to thank my mentor, Dr. Uwe Bergmann, for being a great mentor and choosing me to be apart of the Archimedes project. Special thanks to Martin George for all of our help and support; to the SULI program director, James Lindesay, we could not have asked for a better director; to Helen Quinn, for making this program possible. And I would like to thank the D.O.E for the once in a lifetime opportunity to participate 
in the SULI Program. The Archimedes Project is a group collaboration under the direction of Uwe Bergmann. My summer accomplishments at SLAC are a small piece in the over all picture. Special thanks go to the many technicians, engineers and user support personnel at SSRL because without their help, this project would not be possible.

\section{References}

[1] “Infinite Secrets.” Nova. PBS. September 2003

[2] “X-Ray Fluorescence Spectroscopy.” 2 Aug. 2005 $<$ http://www.amptex.com/xrf.html>

[3] XRF Corporation. "Radiation Detection.” 17 August 2005. <http://www.xrfcorp.com/technology/radiation_detection.html >

[4] http://home.slac.stanford.edu/pressreleases http://www.thewalters.org/archimedes/frame.html http://www.nature.com/news/2005/050516/full/050516-8.html

\section{Tables}




\begin{tabular}{|c|c|c|c|c|c|}
\hline \multicolumn{2}{|c|}{ Distance $10 \mathrm{~mm}$} & \multicolumn{2}{|c|}{ Distance $40 \mathrm{~mm}$} & \multicolumn{2}{|c|}{ Distance $80 \mathrm{~mm}$} \\
\hline speed & 496 & speed & 496 & speed & 830 \\
\hline$x$-num pts & 257 & x-num pts & 1007 & x-num pts & 1204 \\
\hline speed & 595 & speed & 595 & & \\
\hline$x$-num pts & 215 & x-num pts & 840 & & \\
\hline \multirow{2}{*}{\multicolumn{2}{|c|}{ Distance $20 \mathrm{~mm}$}} & sneed & 830 & & \\
\hline & & $\begin{array}{l}\text { Speed } \\
\text { x-num pts }\end{array}$ & 600 & & \\
\hline \multirow[t]{2}{*}{ x-num pts } & 507 & & & & \\
\hline & & \multicolumn{2}{|c|}{ Distance $60 \mathrm{~mm}$} & & \\
\hline speed & 595 & speed & 595 & & \\
\hline$x$-num pts & 424 & $x$-num pts & 1257 & & \\
\hline & & & & & \\
\hline speed & 830 & speed & 830 & & \\
\hline x-num pts & 307 & x-num pts & 905 & & \\
\hline
\end{tabular}

Table1: These are the speeds and x-number of points with different distances.

Formulas

1: Shutter time $\Delta \mathbf{x} \div(\mathbf{v} \div \mathbf{s})-.25$ where $\Delta \mathrm{x}$ is the distance of the scan, $\mathrm{v}$ is the speed, and $\mathrm{s}$ is the device scale for steps in one revolution. $(s=157.48)$

2: Count time

$\mathbf{0 . 0 4} \mathbf{m m} \div \mathbf{( v} \div \mathbf{s})$ where $\mathrm{v}$ is the speed and $\mathrm{s}$ is the device scale For steps in one revolution

3: X-num points

$\Delta \mathbf{x} \div \mathbf{0 . 0 4} \mathbf{m m}+\mathbf{7}$ where $\Delta \mathbf{x}$ is the desired scan distance (this formula is only valid when the speed is 496steps/mm)

Figures

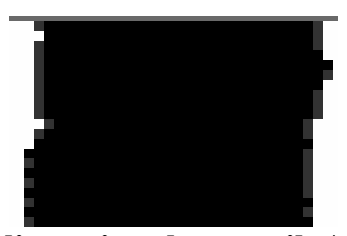

Figure 1: A scan of a line using the stencil. As one can see, neither the left nor the right edge of this line is completely straight. 


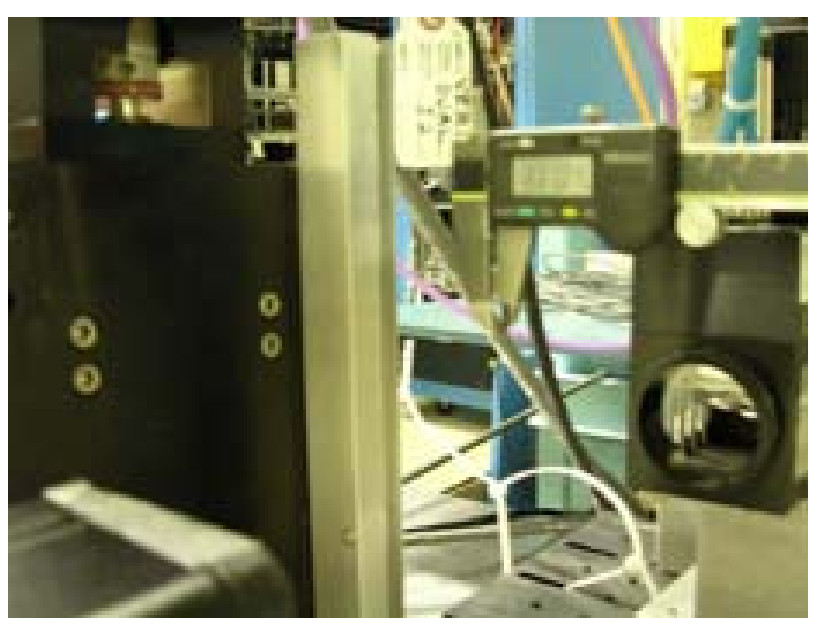

Figure 2: The calipers used to scan

The Oscilloscope

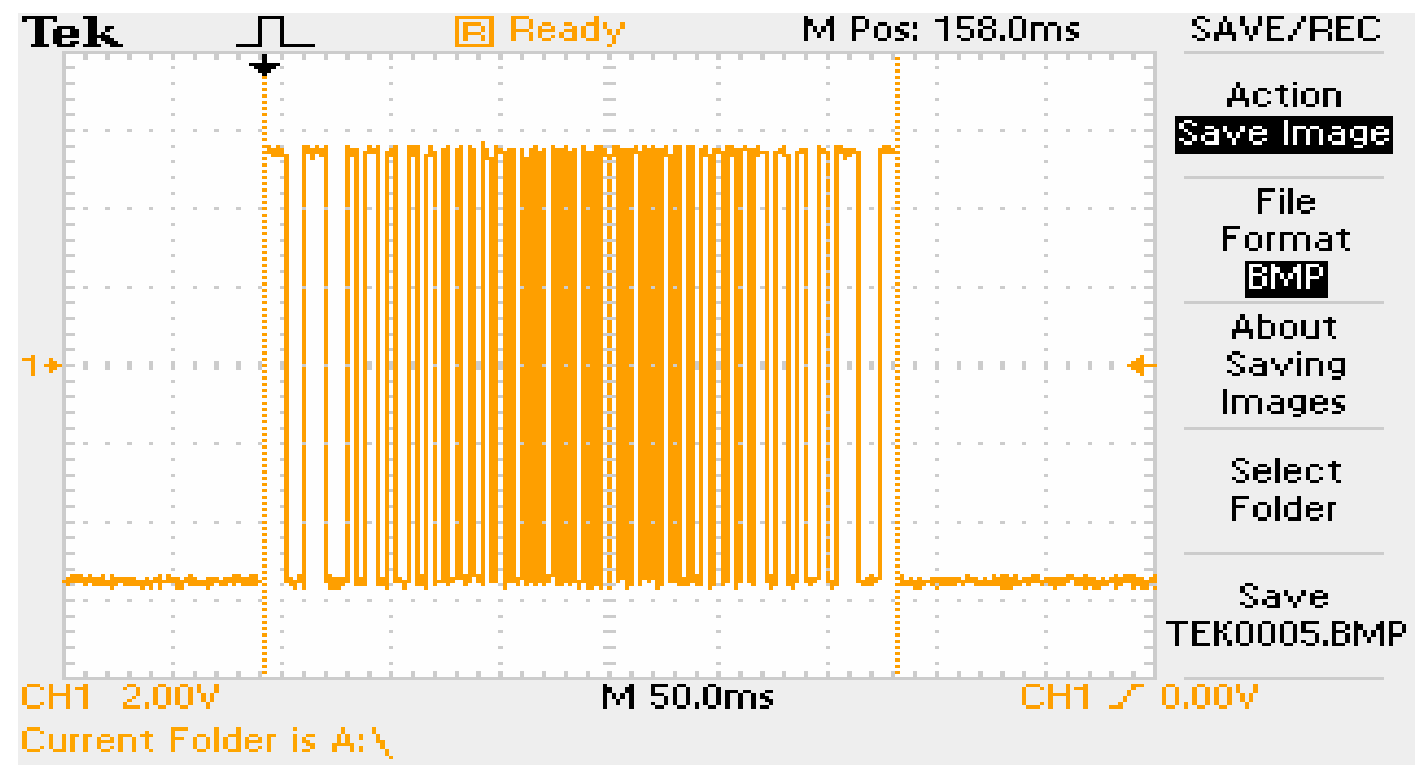

Figure 3: The graph of the motion of the $x$-motor

The graph shows that the motor had constant speed between intervals of

acceleration and deceleration. The reason it is more condensed in the middle is because the graph had to be tightly packed in. 


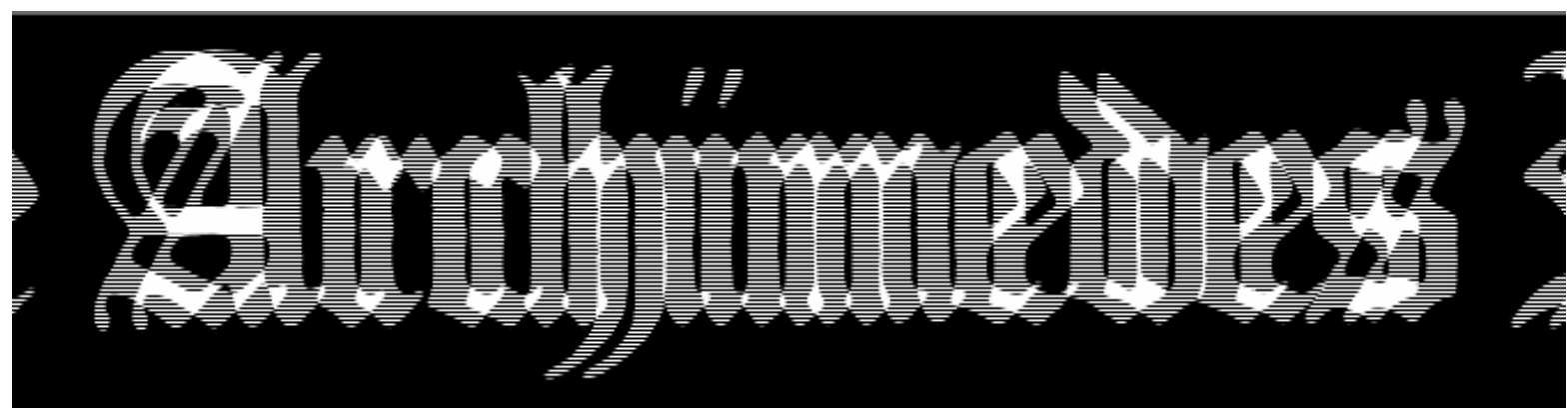

Figure 4: This images was scanned on 08-03-05. Filename: cal-238.ras. The parameters are as follows: Distance $=80, x-$ num pts $=1188$, speed $=830$, accel. $=10,377 \mathrm{dpi}$

This image is considered poor. Subsequent lines do not match up, hence creating a ghost image. It was scanned using the same parameters as the next image, but the $\mathrm{x}$ number of points was changed to 1188.

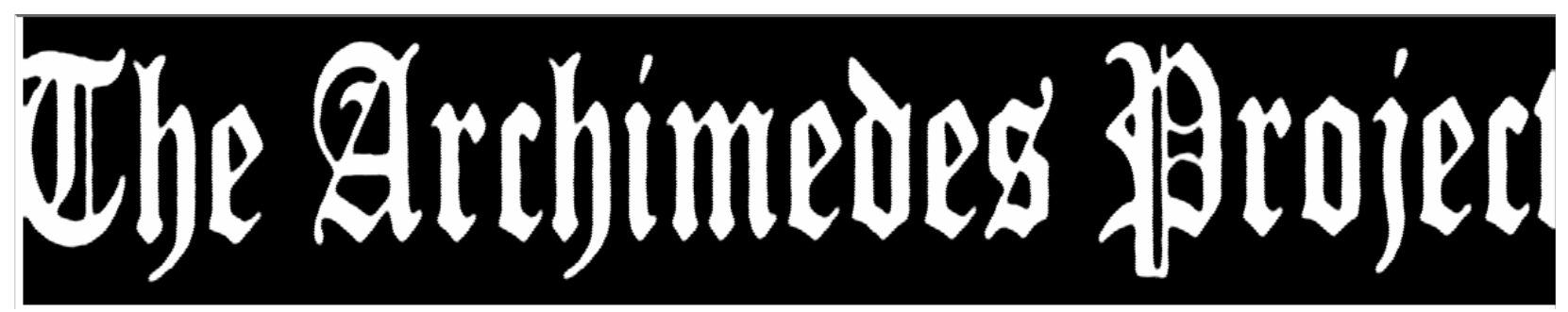

Figure 5: An image scanned on 08-03-05, Filename: cal-229.ras The parameters are as follows: distance $=80 \mathrm{~mm}$, $x$-num pts $=1204$, ppeed $=830$, accel. $=10$, 382dpi

This image is considered to be good. The quality is one pixel per point (relatively small). Subsequent lines match to within one to two pixels. This is shown by a closer look at the characters (see Figure 3). This is the scan of a 18 font text. 


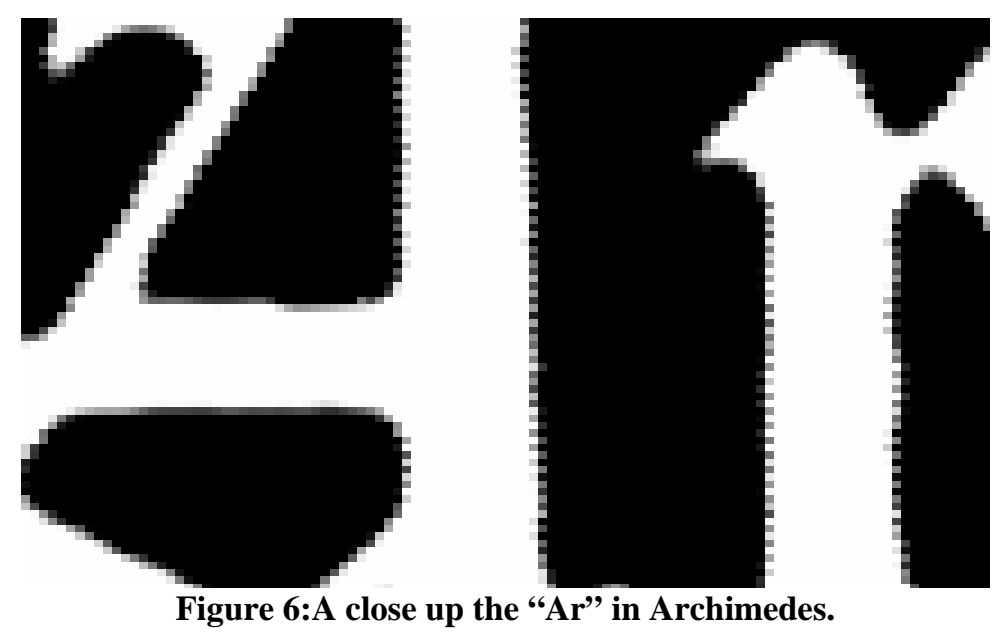

As you can see, the pixels are not in a perfectly straight line; however, the image was taken with the optimal scanning parameters for the distance. 